RESEARCH ARTICLE

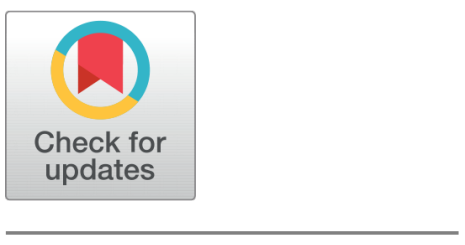

open ACCESS

Received: 07.02.2021

Accepted: 23.04 .2021

Published: 06.05.2021

Citation: Priyokumar SK, Jiten B (2021) Higher Dimensional LRS Bianchi Type-I String Cosmological Model with Bulk Viscosity in General Relativity. Indian Journal of Science and Technology 14(16): 1239-1249. https://doi.org/

10.17485/IJST/v14i16.240

* Corresponding author.

barojiten5@gmail.com

Funding: None

Competing Interests: None

Copyright: (c) 2021 Priyokumar \& Jiten. This is an open access article distributed under the terms of the Creative Commons Attribution License, which permits unrestricted use, distribution, and reproduction in any medium, provided the original author and source are credited.

Published By Indian Society for Education and Environment (iSee)

ISSN

Print: 0974-6846

Electronic: 0974-5645

\section{Higher Dimensional LRS Bianchi Type-I String Cosmological Model with Bulk Viscosity in General Relativity}

\author{
Singh K Priyokumar ${ }^{1,2}$, Baro Jiten ${ }^{1,3 *}$ \\ 1 Department of Mathematical Sciences, Bodoland University, Kokrajhar, 783370, BTR, \\ Assam, India \\ 2 Department of Mathematics, Manipur University, Imphal, 795003, Manipur, India \\ 3 Department of Mathematics, PDUAM, Tulungia, Bongaigaon, 783383, Assam, India
}

\section{Abstract}

Objective: To present a new solution to the field equations obtained for higher dimensional LRS Bianchi type-I universe generated by means of a cloud of strings with particles connected to them with bulk viscosity in general relativity. Methods: To obtain the solutions of field equations of higher dimensional LRS Bianchi type-I universe we consider that the shear scalar of the model is proportional to the scalar expansion of the model $(\sigma \alpha \theta)$, which leads to, $c=b^{\wedge} n$. The physical and geometrical behaviors of the model universe are studied by comparing with the present cosmological scenario and observations. Findings: It is observed that our model is anisotropic, expanding and decelerates at early stage and then accelerates in late universe giving the inflation model universe. Novelty: We obtained new solution to the field equations for higher dimensional LRS Bianchi type-I generated by means of a cloud of strings with bulk viscous fluid in general relativity.

Keywords: LRS Bianchi Typel Metric; Bulk Viscous Fluid; Strings

\section{Introduction}

Still now it is an interesting area for the cosmologists to study and discover the unknown phenomenon of the universe that have yet to observe to study and explore its hidden knowledge. So cosmologists have taken considerable interest to understand the past evolution, present state and evolution in the future of the universe. Before the formation of particles, the strings took major role in the creation and evolution of the universe in early era. Authors in ${ }^{(1,2)}$ initiated the general relativistic study of the strings, and they developed the classical theory of the geometric strings. Because of the major role of strings in describing the evolution of our universe in the early epoch, in recent times, many famous researchers are interested in cosmic strings in general relativity ${ }^{(3,4)}$. Strings give rise to density perturbations leading to the creation of Large Scale Structures (like galaxies) of the universe ${ }^{(5,6)}$ which does not oppose present day observational findings of the universe and results in an anisotropy in the space-time though the strings are unobservable in the present day's universe. These strings possess stress energy and are named as geometric strings and massive strings. After the big-bang explosion there 
was symmetry breaking of the universe spontaneously during the transition of phase in the very early stages of the universe and these cosmic strings which are very important topological defect arose in the early universe as the cosmic temperature went down below some critical point temperatures according to grand unified theories ${ }^{(7-9)}$.

The bulk viscosity assumes an extraordinary part in the development of the early universe. There are numerous events inside the development of the universe wherein the bulk viscosity could emerge. The size of the viscous stress comparative with the expansion is controlled by the coefficients of bulk viscosity. Spatially homogeneous and anisotropic Bianchi typeI models are attempted to comprehend the universe in its beginning phase of the evolution of the universe. The various pictures of the universe may show up at the beginning phase of the cosmological development of the universe because of the dissipative process brought about by viscosity which counteracts the cosmological breakdown (collapse). A few authors endeavored to find the specific solutions of field equations by considering viscous effects in general relativity in isotropic as well as anisotropic cosmological model universes. Authors in ${ }^{(10)}$ have constructed Bianchi type-I cosmological models in presence of bulk viscosity. Bulk viscous fluid Bianchi Type-I string cosmological model in general relativity have been investigated by ${ }^{(11)}$. A new class of LRS Bianchi type-V cosmological model with string dust as a source of gravitational field was investigated in ${ }^{(12)}$. In ${ }^{(13)}$ the authors have endeavored to introduce another solution for the field equations obtained for Bianchi type-III cosmological model in Lyra manifold by utilizing the law of variation of Hubble's Parameter $(\mathrm{H})$, which yields constant DP.

Nowadays it is very interesting to study string cosmology in five-dimensional space-time in general relativity. The possibility of space-time having more than four dimensions (Extra dimensions) has fascinated many researchers. In the recent years, to study cosmological models, the higher dimensional space-time has been given more importance. Generally, the higher dimensional model was introduced by ${ }^{(14)}$ and ${ }^{(15)}$ in an effort to unify gravity with electromagnetism. Higher dimensional model can be regarded as a tool to illustrate the late time expedited expanding paradigm ${ }^{(16)}$. Investigation of higher dimensional space-time can be regarded as a task of paramount importance as the universe might have come across a higher dimensional era during the initial epoch ${ }^{(17)}$. Marciano ${ }^{(18)}$ asserts that the detection of a time varying fundamental constants can possibly show us the proof for extra dimensions. According to ${ }^{(19)}$ and ${ }^{(20)}$, extra dimensions generate huge amount of entropy which gives possible solution to atness and horizon problem. Since we are living in a $4 \mathrm{D}$ space-time, the hidden extra dimension in 5D is highly likely to be associated with the invisible $\mathrm{DM}$ and $\mathrm{DE}{ }^{(21)}$. Several authors have investigated various Bianchi type problems in the field of higher dimensional space-time. An in homogeneous higher dimensional cosmological model with massive string in general relativity was constructed by ${ }^{(22)}$. An LRS Bianchi type-I inflationary string Cosmological model with massive scalar field in general relativity in the field of five-dimensional space-time was constructed by ${ }^{(23)}$ and found that, $\rho+\lambda=0$. In ${ }^{(24)}$ the authors constructed some Bianchi type-III cosmological models with massive string as a source of gravitational field in five-dimensional space-time in general relativity. A Bianchi type-III cosmological models with string in general relativity with magnetic field is obtained by ${ }^{(25)}$, where they used the condition $\rho+\lambda=0$ to solve the field equations. An LRS Bianchi typeI model in cosmology with bulk viscous fluid in Lyra geometry with the help of displacement vector depending upon time was constructed in ${ }^{(26)}$ and found that the bulk viscosity decreases with time. In ${ }^{(27)}$ a perfect fluid cosmological model in Lyra Geometry was studied by using constant deceleration parameter in five dimensional LRS Bianchi type-I Space-time.Considering reasonable cosmological assumptions within the limit of the present cosmological scenario, a spherically symmetric metric in five dimensional setting in the framework of Lyra geometry is analysed ${ }^{(28)}$. A cosmological model in 5D spherically symmetric space-time with energy momentum tensors of minimally interacting fields of dark matter and holographic dark energy in Brans-Dicke theory was constructed by ${ }^{(29)}$. Authors in ${ }^{(30-37)}$ are the authors who studied different string cosmological models in general relativity in different context in various space-times.

Above discussion and investigations motivated us to study the five-dimensional LRS Bianchi type-I string model in cosmology with particles attached to them in general relativity. The paper is presented as: In Section 1, a brief presentation of strings, bulk viscosity and their importance are discussed. In Section 2, the five-dimensional LRS Bianchi type-I metric is introduced and the field equations in the framework of general relativity are determined; In Section 3, using some simplifying assumptions we find the determinate solutions of the survival field equations. In Section 4, we examined the geometrical and physical properties of our model universe with the help of graphs; In Section 5 conclusions are presented.

\section{The Metric and Field Equations}

We consider the 5-dimensional LRS Bianchi type-I metric as

$$
d s^{2}=-d t^{2}+a^{2} d x^{2}+b^{2}\left(d y^{2}+d z^{2}\right)+c^{2} d m^{2}
$$

Here $\mathrm{a}, \mathrm{b}$ and $\mathrm{c}$ are the metric functions of cosmic time $\mathrm{t}$ alone and the extra coordinate "m" is taken to be space-like. 
For the above metric lets

$$
x^{1}=x, x^{2}=y, x^{3}=z, x^{4}=m \text { and } x^{5}=t
$$

In general relativity the Einstein's field equation is written as

$$
R_{i j}-\frac{1}{2} R g_{i j}=-T_{i j}
$$

For a cloud string the energy-momentum tensor is

$$
T_{i j}=\rho u_{i} u_{j}-\lambda x_{i} x_{j}-\xi \theta\left(u_{i} u_{j}+g_{i j}\right)
$$

Where, $\rho$ is the energy density and $\lambda$ the tension density of the string and they are related as $\rho=\lambda+\rho_{p}$, where $\rho_{p}$ is the particle density of matter, $\xi$ is the coefficient of viscosity and $\theta$ is the expansion scalar. The co-ordinates are co-moving, $x^{i}$ is the unit space-like vector indicating the direction of strings and $u^{i}$ is the five velocity vector which satisfies the conditions-

$$
\begin{gathered}
u_{i} u^{i}=-1=-x_{i} x^{i} \\
\text { and } u_{i} x^{i}=0
\end{gathered}
$$

Here without loss of generality we can take

$$
u^{i}=(0,0,0,0,1) \text { and } x^{i}=\left(a^{-1}, 0,0,0,0\right)
$$

The spatial volume is given by

$$
V=a b^{2} c=R^{4}
$$

Where $R(t)$ is the average scale factor of the universe.

The Scalar Expansion is given by

$$
\theta=\frac{\dot{a}}{a}+2 \frac{\dot{b}}{b}+\frac{\dot{c}}{c}
$$

Hubble Parameter is given by

$$
H=\frac{1}{4}\left(\frac{\dot{a}}{a}+2 \frac{\dot{b}}{b}+\frac{\dot{c}}{c}\right)
$$

Deceleration parameter is

$$
q=-\frac{R \ddot{R}}{\dot{R}^{2}}
$$

The shear scalar is given by

$$
\sigma^{2}=\frac{1}{2} \sigma_{i j} \sigma^{i j}=\frac{1}{2}\left[\left(\frac{\dot{a}}{a}\right)^{2}+2\left(\frac{\dot{b}}{b}\right)^{2}+\left(\frac{\dot{c}}{c}\right)^{2}\right]
$$

And the mean anisotropy parameter is given by

$$
\Delta=\frac{1}{4} \sum_{i=1}^{4}\left(\frac{H_{i}-H}{H}\right)^{2}
$$

Where, $\mathrm{H}_{i}(\mathrm{i}=1,2,3,4)$ represents the directional Hubble Parameters in the directions of $\mathrm{x}, \mathrm{y}, \mathrm{z}$ and $\mathrm{m}$ axes and are defined as $H_{1}=\frac{\dot{a}}{a}, H_{2}=H_{3}=\frac{\dot{b}}{b}$ and $H_{4}=\frac{\dot{c}}{c}$ for the metric (1). 
Using the equations (3)-(6), the field equation (2) takes the form

$$
\begin{gathered}
2 \frac{\ddot{b}}{b}+\frac{\ddot{c}}{c}+\frac{\dot{b}^{2}}{b^{2}}+2 \frac{\dot{b} \dot{c}}{b c}=\lambda+\xi \theta \\
\frac{\ddot{a}}{a}+\frac{\ddot{b}}{b}+\frac{\ddot{c}}{c}+\frac{\dot{a} \dot{b}}{a b}+\frac{\dot{a} \dot{c}}{a c}+\frac{\dot{b} \dot{c}}{b c}=\xi \theta \\
\frac{\ddot{a}}{a}+2 \frac{\ddot{b}}{b}+\frac{\dot{b}^{2}}{b^{2}}+2 \frac{\dot{a} \dot{b}}{a b}=\xi \theta \\
2 \frac{\dot{a} \dot{b}}{a b}+\frac{\dot{a} \dot{c}}{a c}+2 \frac{\dot{b} \dot{c}}{b c}+\frac{\dot{b}^{2}}{b^{2}}=\rho
\end{gathered}
$$

Here, the overhead dots mean differentiation with time ' $\mathrm{t}$ '.

\section{Solutions of the Field Equations}

In this part, we intend to derive the solutions of the four highly non-linear independent equations (13)-(16) with 6 unknown variables $\mathrm{a}, \mathrm{b}, \mathrm{c}, \xi, \lambda$ and $\rho$. For deterministic solution we considered the following physical plausible conditions:

We consider that the shear scalar and expansion scalar are proportional, which leads to

$$
c=b^{n}
$$

Here,

The reason of assuming the above condition depends on observations of the velocity red-shift relation for extragalactic sources recommended that the Hubble expansion of the universe is isotropic today to within $30 \%{ }^{(38-40)}$. If H is Hubble constant and $\sigma$ is the shear then the red-shift studies limit $\frac{\sigma}{H} \leq 0.30$. If $\theta$ is expansion scalar, the normal to the spatially homogeneous metric is congruence to the homogeneous hyper-surface that satisfies the condition $\frac{\sigma}{\theta}$ is constant ${ }^{(41)}$.

From (14),(15) by using (17), we get

$$
\frac{\dot{b}}{b}\left[\frac{\ddot{b}}{b}(n+1) \frac{\dot{b}}{b}+\frac{\dot{a}}{a}\right]=0
$$

Using (17) in (14) and comparing with (15), we get $n=1$.

From (17) we get

$$
b=c
$$

Equation (18) yields the following cases:

$$
\text { CaseI }: \frac{\ddot{b}}{b}+2 \frac{\dot{b}}{b}+\frac{\dot{a}}{a}=0, \text { and CaseII }: \frac{\dot{b}}{b}=0
$$

We intend to determine the cosmological models for the above two cases separately.

Casel: $\frac{\ddot{b}}{b}+2 \frac{\dot{b}}{b}+\frac{\dot{a}}{a}=0$

Solving we get,

$$
b(t)=\left[3\left(\int \frac{K}{a(t)} d t+K_{1}\right)\right]^{\frac{1}{3}}
$$


Clearly, the solution are not unique because $\mathrm{b}(\mathrm{t})$ can be obtained for any given $\mathrm{a}(\mathrm{t})$. So for further studies here we consider ${ }^{(42)}$

$$
\frac{\ddot{b}}{b}+2 \frac{\dot{b}}{b}=-\frac{\dot{a}}{a}=k(\text { Cons } \tan t)
$$

Now solving (22) and using (19) we get,

$$
\begin{gathered}
a=k_{1} e^{-k t} \\
b=\left[3\left(\frac{k_{2}}{k} e^{k t}+k_{3}\right]^{\frac{1}{3}}\right. \\
c=\left[3\left(\frac{k_{2}}{k} e^{k t}+k_{3}\right]^{\frac{1}{3}}\right.
\end{gathered}
$$

Here, $k_{1}(\neq 0), k_{2}$ and $k_{3}$ are constants of integrations.

The following metric describes the geometry of the model

$$
d s^{2}=-d t^{2}+k_{1}^{2} e^{-2 k t} d x^{2}+\left[3\left(\frac{k_{2}}{k} e^{k t}+k_{3}\right]^{\frac{2}{3}}\left(d y^{2}+d z^{2}+d m^{2}\right)\right.
$$

The tension density of the string is obtained as

$$
\lambda=\frac{k k_{2} e^{k t}}{\left(\frac{k_{2}}{k} e^{k t}+k_{3}\right)}-k^{2}
$$

The energy density of the string is

$$
\rho=\frac{k_{2}^{2} e^{2 k t}}{3\left(\frac{k_{2}}{k} e^{k t}+k_{3}\right)^{2}}-\frac{k k_{2} e^{k t}}{\left(\frac{k_{2}}{k} e^{k t}+k_{3}\right)}
$$

The particle density is given by

$$
\rho_{p}=\frac{k_{2}^{2} e^{2 k t}}{3\left(\frac{k_{2}}{k} e^{k t}+k_{3}\right)^{2}}-\frac{2 k k_{2} e^{k t}}{\left(\frac{k_{2}}{k} e^{k t}+k_{3}\right)}+k^{2}
$$

From (15) we get

$$
\xi \theta=k^{2}-\frac{k_{2}^{2} e^{2 k t}}{3\left(\frac{k_{2}}{k} e^{k t}+k_{3}\right)^{2}}
$$

The spatial volume is obtained as

$$
V=3 k_{1} e^{-k t}\left(\frac{k_{2}}{k} e^{k t}+k_{3}\right)
$$


The scalar expansion is obtained as

$$
\theta=\frac{k_{2} e^{k t}}{\left(\frac{k_{2}}{k} e^{k t}+k_{3}\right)}-k
$$

Using (32) in (30) we get

$$
\xi=\frac{-k_{2}^{2} e^{2 k t}}{3 k k_{3}\left(\frac{k_{2}}{k} e^{k t}+k_{3}\right)^{2}}-\frac{k_{2}}{k_{3}} e^{k t}-k
$$

The Hubble parameter is obtained as

$$
H=\frac{k_{2} e^{k t}}{4\left(\frac{k_{2}}{k} e^{k t}+k_{3}\right)}-\frac{k}{4}
$$

The deceleration parameter is

$$
q=-\left[\frac{4 k_{2} e^{k t}}{k k_{3}}+1\right]
$$

The shear scalar of the model is

$$
\sigma^{2}=\frac{1}{8}\left[3 k^{2}+\frac{k_{2}^{2} e^{2 k t}}{3\left(\frac{k_{2}}{k} e^{k t}+k_{3}\right)^{2}}+\frac{2 k k_{2} e^{k t}}{\left(\frac{k_{2}}{k} e^{k t}+k_{3}\right)}\right]
$$

3.2 Casell: $\frac{\dot{b}}{b}=0$

Solving it we get,

$$
b=k_{4}
$$

From the equation (19) together with (37) we have,

$$
c=k_{4}
$$

Now using equations (37)-(38) in the field equations (13)-(16) we get,

$$
\lambda+\xi \theta=0
$$

$$
\begin{gathered}
\frac{\ddot{a}}{a}=\xi \theta \\
\rho=0
\end{gathered}
$$

Here $\xi, \lambda, \rho$ and a are four unknowns involved in three equation (39)-(41). To obtain a determinate solution we have assumed following different plausible conditions of equations of state as Geometric String

$$
\begin{gathered}
\rho=\lambda(\text { Geometric String) } \\
\text { And } \rho=(1+\omega) \lambda \text { (p-string) }
\end{gathered}
$$

Where, $\omega>0$ is a constant. 
3.2.1 $\rho=\lambda$ (Geometric String)

This yields,

$$
\begin{gathered}
\rho=\lambda=0 \\
\xi=0 \\
\text { and } a=l t+m
\end{gathered}
$$

Where, $l \neq 0$ and $\mathrm{m}$ are integrating constants.

This case leads to the five-dimensional LRS Bianchi type-I vacuum model universe in Einstein's theory of relativity.

The following metric described the geometry of our model,

$$
d s^{2}=-d t^{2}+(l t+m)^{2} d x^{2}+k_{4}^{2}\left(d y^{2}+d z^{2}+d m^{2}\right)
$$

3.2.2 $\rho=(1+\omega) \lambda$ (p-string)

Using (41) in (43) we get,

$$
(1+\omega) \lambda=0
$$

Which yields either $\omega=-1$ or $\lambda=0$. But $\omega=-1$ is not acceptable as $\omega>0$.

Since, $\lambda=0$ the model in this case also reduces to the model already obtained in above case.

\section{Interpretations of the Results}

In case I we have obtained a five-dimensional LRS Bianchi type-I cosmological model universe with string in general relativity given by (26). The variation of parameters with time for this model are shown below by taking, $k=-1, k_{1}=k_{2}=k_{3}=1$ and the physical and geometrical behavior of the model are discussed as

- At initial epoch $\mathrm{t}=0$, the metric (model) (26) becomes flat.

- It is seen that at time $\mathrm{t}=0$ the evolution of energy density $\rho$ is infinite and it decreases gradually as the time $t$ increases and become constant after some finite time (Figure 1). This model satisfies the conditions $\rho \geq 0$ and $\rho_{p} \geq 0$ (Known as energy density conditions).

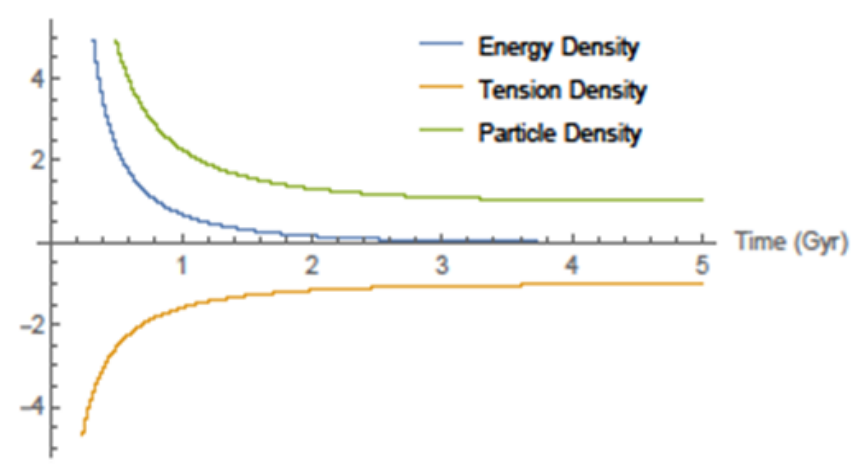

Fig 1. Variation of $\rho, \lambda, \rho_{p}$ Vs. time $\mathrm{t}$ 
- It is also seen from Figure 1 that $\rho>0, \lambda<0$ and $\rho_{p}>0$ showing that at early era particles exists with positive $\rho_{p}$ but strings exist with negative $\lambda$. The particle density $\rho_{p}$ has a large value during the time of big-bang when $t=0$ and as the evolution of time it decreases and moves to a finite value (constant) at $t \rightarrow \infty$, which corresponds to total constant finite number of particles in the late universe. This may correspond to the matter dominated era. From the comparative study of tension density $\lambda$ and particle density $\rho_{p}$ with time as shown in Figure 1, it is seen that $|\lambda|<\left|\rho_{p}\right|$ which describes that in the late time the string vanishes, leaving particles only.

- Initially at $t=0$ the expansion scalar $\theta$ is finite and very large and as the time progresses gradually it decreases and it becomes a small constant after some finite time (as shown in the Figure 2 ) explaining the Big-Bang scenario of the universe. So, this model shows that the universe is expanding with the increase of time but the rate of expansion becomes slower than time increases and the expansion becomes finite at $t \rightarrow \infty$.

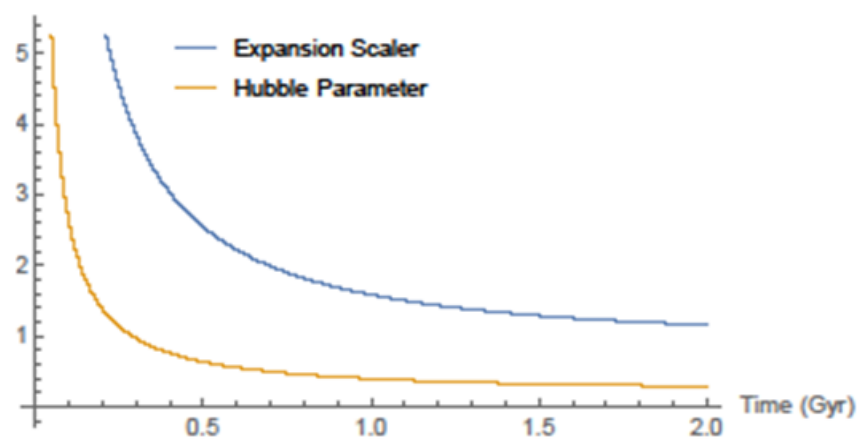

Fig 2. Variation of $\theta, \mathrm{H}$ Vs. time $\mathrm{t}$

- The average Hubble parameter $\mathrm{H}$ is a decreasing function of time. It is large constant when $t \rightarrow 0$ but as the time progresses gradually it also decreases and reached to a small constant value after some finite time as shown by Figure 2 and at $t \rightarrow \infty$, the value of $\frac{d H}{d t} \rightarrow 0$, which also shows the expanding universe of our model.

- Since, $\frac{\sigma^{2}}{\theta^{2}}=$ Constant $(\neq 0)$ as $t \rightarrow \infty$ and hence our model universe obtained here is anisotropic one. Though the anisotropy is included, it does not make any contradiction with the present day observational findings that the universe is isotropic one. This is due to the reason that during the process of evolution of our universe, the initial anisotropy disappears after some epoch and approaches to the isotropy in late time universe.

- From the Figure 3 it is seen that the spatial volume $\mathrm{V}$ is finite at initial epoch $t=0$ and it increases exponentially as $\mathrm{t}$ increases and becomes infinite as $t \rightarrow \infty$, which shows the expanding universe of our model.

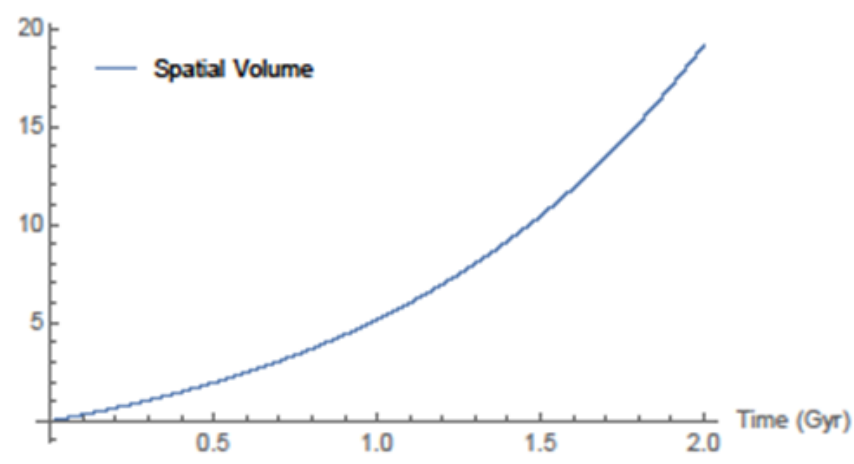

Fig 3. Variation of Volume (V) Vs. time t.

- The DP "q" in the model is decelerating $q>0$ at $t=0$ and it decreases as time increases and becomes accelerating $q<0$ after some finite time as shown in Figure 4. Also $q \rightarrow-1$ at $t \rightarrow \infty$, which indicates that the present model universe 
has a transition from decelerating phase to accelerating phase, indicating the inflation in the model after an epoch of deceleration.

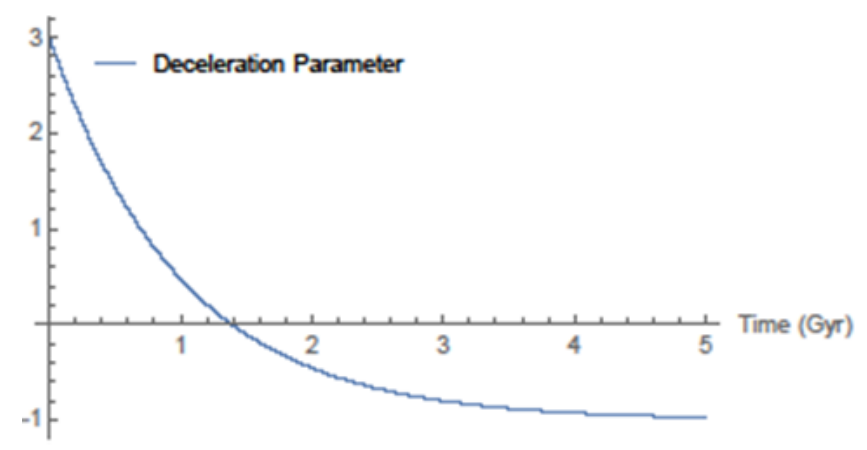

Fig 4. Variation of DP (q) Vs. time t.

- The bulk viscosity for this model is decreasing function of time. The function of the bulk viscosity is to retard the expansion of the universe and since bulk viscosity deceases with the time so retardness also decreases which supports in the expansion in faster rate in the late time universe. From the above discussion it can be seen that the bulk viscosity plays a great function in the evolution of the universe.

The case II, leads to LRS Bianchi type-I vacuum model in Einstein's theory of relativity in five-dimensional space-time represented by which is not realistic because strings don't survive for this model.

\section{Conclusions}

In this study, we have investigated a five-dimensional LRS Bianchi type-I String cosmological model in general theory of relativity in presence of bulk viscous fluid given by (26) which is an inflationary model. The model universe obtained here is anisotropic, accelerating and expanding. The DP "q" obtained here is decelerating at initial stage and accelerates after some finite time, indicating inflation in the model after an epoch of deceleration which is in accordance with the present day observational scenario of the accelerated expansion of our universe as type la supernovae ${ }^{(43,44)}$. The model universe obtained here is anisotropic one in the early epoch but the recent observations tell us that there is disparity in the measurement of intensities of microwaves coming from the sky in different directions, which urge us to study about the universe with LRS Bianchi type-I metric to describe the universe in more reasonable circumstances. During the inflation, shear decreases with time and then it turns into an isotropic universe with very small shear. As expected our model satisfies the energy conditions $\rho \geq 0$ and $\rho_{p} \geq 0$ which in turn imply that our derived models are physically realistic as the present day observational data. The tension density and the particle density are comparable and the model represents a matter dominated universe in the late time which agrees with the present-day observational findings. Also, the model represents an exponentially expanding universe that starts with big-bang at cosmic time $t=0$ giving inflationary model. The bulk viscosity is the decreasing function of time (t). Thus, our model universe has cosmological importance since it is clarifying the early universe and it should be a sensible representation of the universe at early age.Finally, we may conclude that all the above solutions and results presented in the paper are new, are in good agreement with the present-day cosmological observations and useful for a better understanding of the evolution of the universe in Bianchi type-I space-time with bulk viscous fluid. This study is likely to be useful for the analysis of different kinds of Bianchi Models in various space-times. The model needs further deep study in higher dimensional space-time considering all the observational findings, which will be our upcoming work.

\section{References}

1) Stachel J. Thickening the string. I. The string perfect dust. Physical Review D. 1980;21(8):2171-2181. Available from: https://dx.doi.org/10.1103/physrevd. 21.2171.

2) Letelier PS. String cosmologies. Physical Review D. 1983;28(10):2414-2419. Available from: https://dx.doi.org/10.1103/physrevd.28.2414.

3) Kibble TWB. Topology of cosmic domains and strings. Journal of Physics A: Mathematical and General. 1976;9(8):1387-1398. Available from: https://dx.doi.org/10.1088/0305-4470/9/8/029. 
4) Kibble TWB. Some implications of a cosmological phase transition. Physics Reports. 1980;67(1):183-199. Available from: https://dx.doi.org/10.1016/ 0370-1573(80)90091-5.

5) Ya BZ, Yu IK, Okun LB. Cosmological Consequences of the Spontaneous Breakdown of Discrete Symmetry. Zh Eksp Teor Fiz. 1974;67:3-11. Available from: http://www-public.slac.stanford.edu/sciDoc/docMeta.aspx?slacPubNumber=SLAC-TRANS- 0165 .

6) Ya BZ. Cosmological fluctuations produced near a singularity. Mon Not R AstronSoc. 1980;192(4):663-667. Available from: 10.1093/mnras/192.4.663.

7) Everett AE. Cosmic strings in unified gauge theories. Physical Review D. 1981;24(4):858-868. Available from: https://dx.doi.org/10.1103/physrevd.24.858.

8) Vilenkin A. Cosmic strings. Physical Review D. 1981;24(8):2082-2089. Available from: https://dx.doi.org/10.1103/physrevd.24.2082. doi:10.1103/physrevd.24.2082.

9) Vilenkin A. Gravitational field of vacuum domain walls and strings. Physical Review D. 1981;23(4):852-857. Available from: https://dx.doi.org/10.1103/ physrevd.23.852.

10) Banerjee A. String Cosmology in Bianchi I Space-Time. Pramana J Phys. 1990;34:1-11.

11) Humad V, Nagar H, Shrimali S. Bulk Viscous Fluid Bianchi Type-I String Cosmological Model in General Relativity. IOSR Journal of Mathematics. 2016;12:11-15. Available from: 10.9790/5728-1202041115.

12) Dubey RK, Srivastava SK. A new class LRS Bianchi type-V string dust cosmological model in modified general relativity. IJRAT. 2018;6:3684-3686.

13) Baro J, Singh PK, Singh TA. Mathematical analysis on anisotropic Bianchi Type-III inationary string Cosmological models in Lyra geometry. Indian Journal of Science and Technology. 2021;14(1):46-54. Available from: https://doi.org/10.17485/IJST/v14i1.1705.

14) Kaluza T. Zum unitätsproblem der physik Sitzungsber. Preuss Akad Wiss Berlin Math Phys. 1921;22:966.

15) Klein O. Quantentheorie Und Fünfdimensionale Relativitätstheorie. Z Physik. 1926;37:895-906.

16) Banik S, Bhuyan K. Dynamics of higher-dimensional FRW cosmology in $R \mathrm{p} \exp (\lambda \mathrm{R})$ gravity. Pramana. 2017;88(2):26. Available from: https: //dx.doi.org/10.1007/s12043-016-1335-2.

17) Singh GP, Deshpande RV, Singh T. Higher-dimensional cosmological model with variable gravitational constant and bulk viscosity in Lyra geometry. Pramana. 2004;63(5):937-945. Available from: https://dx.doi.org/10.1007/bf02704332.

18) Marciano WJ. Time Variation of the Fundamental "Constants" and Kaluza-Klein Theories. Phys Rev Lett . 1984;52(7):489-491. Available from: https://doi.org/10.1103/PhysRevLett.52.489.

19) Alvarez E, Gavela MB. Entropy from Extra Dimensions. Physical Review Letters. 1983;51(10):931-934. Available from: https://dx.doi.org/10.1103/ physrevlett.51.931.

20) Guth AH. Inflationary universe: A possible solution to the horizon and flatness problems. Physical Review D. 1981;23(2):347-356. Available from: https://dx.doi.org/10.1103/physrevd.23.347.

21) Chakraborty S, Debnath U. Higher Dimensional Cosmology with Normal Scalar Field and Tachyonic Field. International Journal of Theoretical Physics. 2010;49(8):1693-1698. Available from: https://dx.doi.org/10.1007/s10773-010-0348-8.

22) Chatterjee S. Massive strings in higher-dimensional inhomogeneous spacetime. General Relativity and Gravitation. 1993;25(10):1079-1087. Available from: https://dx.doi.org/10.1007/bf00763555.

23) Mohanty G, Samanta GC. Five dimensional String Cosmological models with massive Scalar field. FIZIKA B. 2010;19:43-52.

24) Samanta GC, Biswal SK, Mohanty G. Some five dimensional Bianchi type-III String Cosmological models in General Relativity. Bulg J Phys. 2011;38:380389.

25) Kandalkar SP, Samdurkar SW, Gawande SP. Bianchi Type-III String Cosmological Models in The Presence of Magnetic Field in General Relativity. Int $J$ Sci Eng Res. 2012;3(11):1-7. Available from: https://www.ijser.org/researchpaper/Bianchi-Type-III-String-Cosmological-Models-in-The-Presence-ofMagnetic-Field-in-General-Relativity.pdf.

26) Kandalkar SP, Samdurkar S, Bianchi L. Type I Cosmological Model with Bulk Viscosity in Lyra Geometry. Bulg J Phys. 2015;42:42-52.

27) Singh PK, Mollah MR. Higher dimensional LRS Bianchi type-I Cosmological model universe Interacting with perfect fluid in Lyra Geometry. The African Review of physics. 2016;11:33-38.

28) Singh PS, Singh KP. A higher dimensional cosmological model for the search of dark energy source. IJGMMP. 2020;18:2150026.

29) Singh KP, Singh P. Dark energy on higher dimensional spherically symmetric Brans-Dicke universe. Chinese JPhys. 2019;60:239-247. Available from: https://doi.org/10.1016/j.cjph.2019.05.003.

30) Singh CP. String cosmology with magnetized bulk viscous fluid in Bianchi I universe. Astrophysics and Space Science. 2013;343(2):773-781. Available from: https://dx.doi.org/10.1007/s10509-012-1236-x.

31) Khadekar GS, Patki V, Radha R. String dust cosmological model in higher- dimensional space time. International Journal of Modern Physics D. 2005;14(09):1621-1634. Available from: https://dx.doi.org/10.1142/s0218271805007292.

32) Khadekar GS, Tade SD. String cosmological models in five dimensional bimetric theory of gravitation. Astrophysics and Space Science. 2007;310(1-2):4751. Available from: https://dx.doi.org/10.1007/s10509-007-9410-2.

33) Khadekar GS, Patki VA, Radha R. String Cosmological Model with Bulk Viscosity in Higher Dimensional Space Time. Journal of Dynamical Systems and Geometric Theories. 2007;5(2):117-123. Available from: https://dx.doi.org/10.1080/1726037x.2007.10698531.

34) Yadav AK, Yadav VK, Yadav L. Bianchi Type-V String Cosmological Models in General Relativity. Pramana. 2011;76(4):681-690.

35) Tripathi B, Tyagi A, Parikh S. Bianchi Type-I Inhomogeneous String Cosmological Model with Electromagnetic Field in General relativity. Prespacetime J. 2017;8(4):474-483.

36) Singh KP, Baro J. Bulk Viscous Fluid Bianchi Type-I string Cosmological Model with Negative Constant Deceleration Parameter. Advances in Mathematics: Scientific Journal. 2020;9(7):4907-4916. Available from: https://dx.doi.org/10.37418/amsj.9.7.55.

37) Baro J, Singh KP. Higher Dimensional Bianchi Type-III String Universe With Bulk Viscous Fluid and Constant Deceleration Parameter(DP). Advances in Mathematics: Scientific Journal. 2020;9(10):8779-8787. Available from: https://dx.doi.org/10.37418/amsj.9.10.101.

38) Thorne KS. Primordial Element Formation, Primordial Magnetic Fields, and the Isotropy of the Universe. The Astrophysical Journal. 1967;148:51-51. Available from: https://dx.doi.org/10.1086/149127.

39) Kantowski R, Sachs RK. Some Spatially Homogeneous Anisotropic Relativistic Cosmological Models. Journal of Mathematical Physics. 1966;7(3):443-446. Available from: https://dx.doi.org/10.1063/1.1704952.

40) Kristian J, Sachs RK. Observations in Cosmology. The Astrophysical Journal. 1966;143:379-379. Available from: https://dx.doi.org/10.1086/148522.

41) Collins CB, Glass EN, Wilkinson DA. Exact spatially homogeneous cosmologies. General Relativity and Gravitation. 1980;12(10):805-823. Available from: https://dx.doi.org/10.1007/bf00763057. 
42) Mohanty G, Mahanta KL. Five-dimensional axially symmetric string cosmological model in Lyra manifold. Astrophysics and Space Science. 2007;312(34):301-304. Available from: https://dx.doi.org/10.1007/s10509-007-9691-5.

43) Riess A. Observational evidence from Supernovae for an accelerating Universe and a Cosmological Constant. Astron J. 1998;116:1-36.

44) Perlmutter S. Mesurments of $\Omega$ and $\Lambda$ from 42 high-redshift supernovae. Astrophys J. 1999;517:565-586. 